\title{
MANIFESTATION OF SLEEP BRUXISM ACCORDING TO THE AGE OF PATIENTS Dumitru Romaniuc ${ }^{1 a^{*}}$, Valeriu Fala ${ }^{1 b}$, Victor Lacusta ${ }^{2 c}$, Gheorghe Bordeniuc ${ }^{1 a}$, Paula Fala ${ }^{3 \mathrm{~d}}$
}

\author{
'Department of Therapeutic Dentistry, ${ }_{\prime \prime}$ Nicolae Testemițanu"S State University of Medicine and Pharmacy, Chişinău, Republic of Moldova \\ ${ }^{2}$ Department of Alternative and Complementary Medicine, \\ ${ }^{3}$ Institute of Neurology and Neurosurgery, Chişinău, Republic of Moldova
}

\author{
aphD Fellow \\ bDDS, PhD, MSc, Assoc. Prof. \\ PhD, Univ. Prof., Academician \\ ${ }^{\mathrm{d}}$ Neurology resident
}

\section{ABSTRACT}

DOI: 10.25241/stomaeduj.2018.5(1).art.3

\begin{abstract}
Introduction: Bruxism is a widespread condition, affecting up to $85-90 \%$ of the general population, and in $5 \%$ of these individuals, the grinding evolves into a clinical condition. In many cases, sleep bruxism is latent, the disorder may be identified by its consequences (dental wear, etc.). This ascertains the need to develop diagnostic methods that may be able to identify bruxism at the initial stages of the disorder.
\end{abstract}

Aim of the study: to determine the peculiarities of the clinical manifestation of primary sleep bruxism (SB) based on the age of the patients.

Methodology: One hundred patients with primary SB (70 patients aged between 1835 and 30 patients aged between 35-50) were investigated. The clinical features of SB (algic syndrome, myogenic disorders, temporomandibular disorders, dental wear, psychoemotional disorders, sleep quality alterations) were also investigated.

Results: The expression of emotional stress in patients of various ages was almost identical. The highest clenching frequency and duration, as well as a higher clinical expression of sleep bruxism was observed in patients under 35. The components of the algic syndrome show a varied expression for different age groups - patients under 35 had more severe disorders of the nocturnal episodes and myogenic-spastic events; older patients (35-50) were characterized by a prevalence of local and diffuse myogenic structural disorders, arthrogenic pain and severe sleep disturbances.

Conclusion: Electromyography and ultrasonography can reveal important morphofunctional peculiarities of the masticatory muscles that are associated with the severity of SB in patients from different age groups.

Keywords: bruxism, electromyography, ultrasonography, temporomandibular disorders, diagnostics.

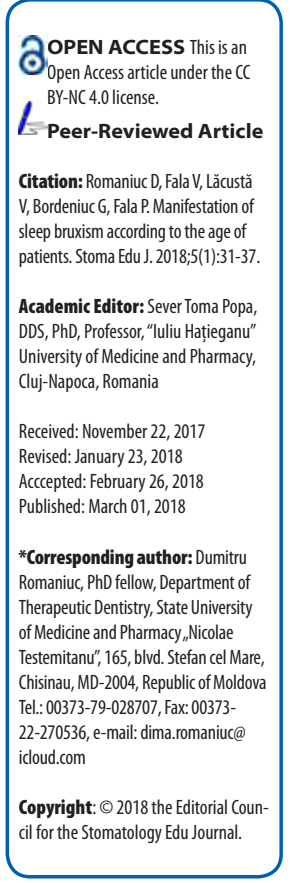

\section{Introduction}

Bruxism is a parafunctional activity, characterized by repetitive jaw-muscle clenches, tooth grinding, bracing/thrusting of the jaw, occurring either during sleep (SB - sleep bruxism) or during wakefulness (AB awake bruxism) [1]. Bruxism is a widespread condition - around $85-90 \%$ of the general population grinds their teeth in certain periods of their life, and in $5 \%$ of these people, the grinding evolves into a clinical condition $[2,3]$. The prevalence of bruxism varies greatly from $5 \%$ to $96 \%$, which highlights that a varied range of criteria and methods for diagnosing this condition are being used [5,6]. An important aspect to this problem is the age of the patients. The highest frequency of bruxism is observed in the age group between 19-44, without any essential gender- based differences [7]. Bruxism may be observed in children starting approximately from the age of one [8] and it occurs in 7-15\% of cases [9]. The condition has its onset during childhood in $6.4 \%$ of cases, during school years in $2.5 \%$ of cases and during the period of professional activity in $26.8 \%$ of cases [10]. The prevalence of bruxism in children varies from $6.5 \%$ to $28 \%$ in various countries $[11,12]$. The authors, based on the analysis of various scientific literature data, have established a linear trend of decrease in the prevalence of bruxism in relation to age: $19 \%$ at the age of $3-10$,
$13 \%$ in adolescents and youngsters, $3 \%$ in adults after the age of 60 [11]. Some researchers consider that bruxism first identified in childhood may continue to persist with aging [13], others have established that bruxism diminishes and disappears during adulthood [14]. The incidence of bruxism in 18-29 year-olds is $18.9 \%$ - awake bruxism in $10.4 \%$ of cases, sleep bruxism in $2.4 \%$ of cases and mixed bruxism in $6.1 \%$ of cases. It has been established that patients aged between 20-29 show signs of bruxism most frequently (41.8\%) [15]. An analysis of 2,000 people showed that bruxism is present in 18-year-olds in $13 \%$ of cases, and after 65 years of age in only $3 \%$ at an approximately equal frequency in men and women [16]. After the age of 65, the prevalence of bruxism is about $10 \%$ with a dramatic decrease with aging. The prevalence of bruxism in individuals aged between 35-44 is twice as high as the prevalence in 18-year-olds, respectively $4.12 \pm 0.79 \%$ vs. $2.73 \pm 0.65 \%$. Currently, there are no specific factors that are deemed responsible for the etiology of bruxism [4]. It is being assumed that one of the causes of the higher prevalence of night bruxism in young people is the presence of a higher level of anxiety and stress [11]. With aging, the microstructure of the masticatory muscles essentially changes; signs of osteoporosis that are associated with the changes in the masticatory 
muscles occur [17]. The thickness and hypertrophy of the masticatory muscles also depend on the age of the patients $[18,19,20]$. Based on the age factor, the degree of severity is also various for the manifestations of sleep bruxism. It is being assumed that the peculiarities of bruxism based on the age factor are manifested under the influence of cofactors (alcohol, caffeine, nicotine, etc.), professional activity, and especially stressful professions [6,12]. Currently, there are no sufficient studies on the peculiarities of SB manifestation based on the age of the patients, while the etiopathogenetic causes of this phenomenon are not yet clear. A clinicalneurophysiological study of bruxism at various periods of life will allow the development of a diagnostic and a treatment algorithm based on the age of the patients. Clinical signs and the subjective symptoms of sleep bruxism are considered to be neither always present, nor evident [4]. In many cases, sleep bruxism is latent (subclinical), without being revealed during common dental exam. In the subclinical variants of sleep bruxism, the disorder is identified when various complications arise (dental abrasion, root fractures, etc.). This ascertains the need of developing diagnostic methods that may be able to identify bruxism in patients of various ages at the initial stages of the disorder, using pathogenetically argumented methods.

Purpose of the study - highlighting the peculiarities of the clinical manifestations of primary sleep bruxism according to the age of the patients.

\section{Materials and methods}

The study has been approved by the Committee of Research Ethics of the "Nicolae Testemițanu" State University of Medicine and Pharmacy, Chişinău, Republic of Moldova (minutes no. 37/04.04.2016).

One hundred patients with primary sleep bruxism were investigated (Table 1).

The diagnosis of SB was performed following the current international criteria, including: 1) medical history, 2) application of clinical questionnaires, 3) dental clinical examination (intraoral and extraoral), 4) EMG assessment for a period of several days, 5) recording of the SB nocturnal episodes for a period of several nights, 6) ultrasonographic examination of the masticatory muscles.

Inclusion criteria: a positive clinical diagnosis of primary sleep bruxism, confirmed by an objective recording of the nocturnal episodes; occlusal type - Angle Class l; age of patients between 18-50; presence of the patient's consent to participate in the research; cooperating patients.

Exclusion criteria: age was not within the established limits; other clinical forms of bruxism (disorders of the nervous system - epilepsy, parkinsonism, etc.); the presence of anomalies and inflammatory signs in the stomatognathic system; the presence of signs of organic damage with the decompensation of the masticatory activity (EMG values during relaxation less than $30 \mu \mathrm{V}$ ); various acute and chronic diseases in the period of exacerbation, parasitosis; alcoholism, drug addiction, toxicomania, mental illness; ongoing treatment with psychotropic, anticonvulsant or miorelaxant drugs; lack of the patient consent for participating in the research;
Table 1. The age of the patients with primary sleep bruxism according to their gender.

\begin{tabular}{rrrr}
\hline \multirow{2}{*}{$\begin{array}{r}\text { Researched } \\
\text { groups }\end{array}$} & $\begin{array}{r}\text { Mean age of } \\
\text { the group, }\end{array}$ & \multicolumn{2}{r}{ Mean age, yrs. } \\
\cline { 3 - 4 } & yrs. & $\begin{array}{r}\text { Individuals } \\
\text { under 35 }\end{array}$ & $\begin{array}{r}\text { Individuals } \\
\text { over 35 }\end{array}$ \\
\hline Whole group & $33.2 \pm 1.15$ & $26.6 \pm 0.99$ & $39.8 \pm 1.07$ \\
Women & $30.4 \pm 1.34$ & $23.5 \pm 1.02$ & $37.3 \pm 1.16$ \\
Men & $36.0 \pm 1.12$ & $29.7 \pm 1.25$ & $42.3 \pm 2.13$ \\
\hline
\end{tabular}

non-cooperating patients.

The study regarding the influence of stressogenic professions on the degree of sleep bruxism manifestations was performed according to the criteria proposed by Nishimura [21].

We have applied questionnaires for the quantitative assessment of SB manifestations [22-25]: clinical questionnaire, Fonseca questionnaire, multifactorial questionnaire for bruxism associated pain, sleep quality questionnaire. For the quantitative assessment of the emotional subjective feelings, we have used the Visual Analogue Scale (VAS).

The dental abrasion degree was assessed, according to the following score [22]: 0 - no abrasion; 1 - dental abrasion within enamel boundaries; 2 - dental abrasion with crown destruction less than one-third; 3 - dental abrasion with crown destruction more than one third. In order to record the nocturnal motor episode, we have

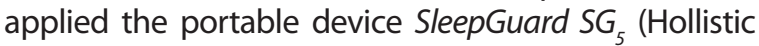
Inc., USA). It is used for five consecutive nights in order to assess the average values of the motor indices: total clench time (TCT) during sleep and their number (TNC - total number of clenches).

We have determined the thickness of the masseter muscle following the recommendations in the scientific literature $[18,19]$ by applying the Envisor C (Philips, Japan) equipment and a 7.5 MHz linear transducer. The masseter muscle thickness gradient (MTG) was calculated according to the formula:

$$
M T G=\frac{G a-G r}{G a} \times 100 \%
$$

where: $G r$-masseter muscle thickness during relaxation (mm); Ga-masseter muscle thickness during maximum jaw engagement ( $\mathrm{mm}$ ).

We have determined the echo-structure of the masseter muscle by analyzing the local and diffuse echogenic features. Normally, the muscle is visualized as hyperechogenic strips parallel to the long axis of the muscle. Muscle fascicles have a connective tissue coating that creates an ultrasonographic picture of hyperechogenic bands. Any pathological change in muscles, fascia, and connective tissue manifests itself through local and/or diffuse modifications of echogenicity. The trigger points are manifested by the occurrence of local pathological echogenicity, and the pathological changes in the masseter muscle can be manifested through diffuse ecogenic zones that increase, consequent to the increase in muscle hypertrophy/hypotrophy, increase in the processes of fibrosis, etc. For the quantitative assessment of the echogenic features, we have determined the total score: normal echogenicity in the projection of the masseter muscle - 0 points; local pathological echogenicity - 1 
I Table 2. Clinical manifestations of primary sleep bruxism vs. the age of patients vs. indices observed in healthy subjects.

\begin{tabular}{|c|c|c|c|c|c|c|}
\hline \multirow[b]{2}{*}{ Indices } & \multirow[b]{2}{*}{ Healthy $(n=30)$} & \multicolumn{3}{|c|}{ Primary sleep bruxism } & \multirow[b]{2}{*}{$P_{1-2}$} & \multirow[b]{2}{*}{$\mathbf{P}_{3-4}$} \\
\hline & & $\begin{array}{l}\text { Entire group } \\
\qquad(n=100)\end{array}$ & $\begin{array}{r}\text { Age } \leq 35 \\
(n=70)\end{array}$ & $\begin{array}{r}\text { Age }>35 \\
(n=30)\end{array}$ & & \\
\hline $\begin{array}{r}\text { Bruxism } \\
\text { questionnaire, points }\end{array}$ & $0.10 \pm 0.05$ & $4.85 \pm 0.28$ & $5.41 \pm 0.32$ & $4.25 \pm 0.24$ & $<0.001$ & $<0.05$ \\
\hline $\begin{array}{r}\text { Pain } \\
\text { questionnaire, points }\end{array}$ & $1.18 \pm 0.16$ & $4.71 \pm 0.25$ & $5.09 \pm 0.36$ & $4.33 \pm 0.31$ & $<0.001$ & NS \\
\hline $\begin{array}{r}\text { Fonseca } \\
\text { questionnaire, points }\end{array}$ & $1.16 \pm 0.39$ & $52.02 \pm 4.13$ & $59.43 \pm 4.42$ & $44.61 \pm 3.75$ & $<0.001$ & $<0.05$ \\
\hline $\begin{array}{l}\text { Ultrasound, } \\
\text { MTG index, \% }\end{array}$ & $25.0 \pm 0.45$ & $28.4 \pm 0.52$ & $25.5 \pm 0.55$ & $31.3 \pm 0.58$ & $<0.001$ & $<0.001$ \\
\hline $\begin{array}{r}\text { Ultrasound, } \\
\text { echogenicity, units }\end{array}$ & $0.23 \pm 0.07$ & $1.35 \pm 0.10$ & $1.10 \pm 0.11$ & $1.60 \pm 0.10$ & $<0.001$ & $<0.01$ \\
\hline Dental wear, units & $0.26 \pm 0.11$ & $1.93 \pm 0.19$ & $1.87 \pm 0.22$ & $1.99 \pm 0.15$ & $<0.001$ & NS \\
\hline
\end{tabular}

Note: NS - no statistical significance.

point; diffuse pathological echogenicity -2 points. The results obtained were processed using the Statistics for Windows v.11.0 (StatSoft Inc., USA) specialized software package for statistical analysis.

\section{Results}

In healthy people, highly stressful professions accounted for $30 \%$ of cases; VAS-stress expression of $3.80 \pm 0.35$ points. In patients with SB, but from various age groups (18-50 years, $n=100)$, highly stressful occupations accounted for $24 \%$ of cases; VAS-stress $5.71 \pm 0.31$ points. In SB patients under 35 , high stress professions accounted for $21.4 \%$ of cases; VAS-stress - $5.99 \pm 0.28$ points. In SB patients over 35, highly stressful occupations accounted for $30 \%$ of cases; VASStress $-5.43 \pm 0.35$ points.

This analysis revealed non-essential differences $(p>$ 0.05 ) between healthy individuals and SB patients of various age. Statistical differences of VAS-stress scores among healthy individuals vs. patients with $S B(n=$ $100)$ were significant $(p<0.01)$, and the differences between groups of patients with SB of various age were not significant $(p>0.05)$ with tendencies of more severe stress manifestation in patients under 35 .

TNC indices in healthy individuals were $3.4 \pm 0.95$; in patients with $S B(n=100)-50.4 \pm 5.96$; in patients with SB under $35-65.4 \pm 5.26$; in patients with SB over 35 $-35.4 \pm 6.44$. The highest frequency of clenching was observed in younger patients.

TCT indices in healthy subjects constituted $3.6 \pm 0.68$; in patients with $S B(n=100)-86.5 \pm 7.93$; in patients with SB under $35-110.5 \pm 8.22$; in patients with SB over 35 $61.5 \pm 6.91$. In a similar way, the highest clench duration was observed in younger patients.

The length and quality of night sleep were closely related to the intensity of the psycho-emotional state. Sleep duration in patients under 35 (18-35 years of age, $\mathrm{n}=70$ ) showed statistically significant differences in comparison to the values observed in older patients (35-50 years, $\mathrm{n}=30$ ), respectively: $7.9 \pm 0.52$ hours vs.
$6.2 \pm 0.41$ hours $(p<0.05)$.

The quality of nighttime sleep in patients with $S B$, based on the self-assessment scores, was as follows (statistically significant differences between values observed in patients under 35 years of age in comparison to older patients):

- normal sleep quality - 20 patients (28.6\%)/4 patients (13.3\%), p > 0.05;

- borderline disorders - 38 patients (54.3\%)/8 patients $(26.7 \%), p<0.01$;

- pathological sleep - 12 patients $(17.1 \%) / 18$ patients $(60.0 \%), \mathrm{p}<0.001$.

The analysis of the clinical manifestations of $\mathrm{SB}$, based on the clinical questionnaire has shown a higher value of the clinical SB expression in patients under 35, when compared to older patients (Table 2). The frequency of the positive responses, based on the SB questionnaire (which reflected the condition of the patients in the last 6 months) was the following (the percentages of clinical signs for patients under 35 are indicated in a decreasing order, as compared to data from older patients):

- Tooth grinding during sleep, observed by life partners $-100 \%$ vs. $100 \%$.

- Tooth grinding during sleep (self-assessed by patients) $-97.1 \%$ vs. $93.3 \%$ ( $p>0.05$ ).

- Pain and fatigue in masticatory muscles, after awakening $-60.0 \%$ vs. $36.7 \%(p<0.05)$.

- Sensations of jaw tension or difficulties during mouth opening, after awakening $-54.3 \%$ vs.

- $30 \%(p<0.05)$.

- Pain felt in the temple region, after awakening $50.0 \%$ vs. $26.7 \%(p<0.05)$.

- Teeth clenched together, after awakening $-44.3 \%$ vs. $23.3 \%(p<0.05)$.

- Dental or gingival pain, after awakening $-22.9 \%$ vs. $10.0 \%(p>0.05)$.

- Dental mobility, after awakening - $18.6 \%$ vs. $13.3 \%(p>0.05)$.

In patients with SB under 35, ulcerations of the tongue were detected in $7.1 \%$ of cases vs. $6.7 \%$ in patients 
aged over 35.

According to the multifactorial pain test developed by the authors, the smallest values in healthy individuals have the following scales: scale I - arthrogenic factor $(0.10 \pm 0.05)$, autonomous factor, scale IV $(0.10 \pm$ $0.15)$, circadian factor, scale $\mathrm{VI}(0.10 \pm 0.05)$ and the pain duration factor, scale VIII $(0.10 \pm 0.05)$. In healthy individuals, the highest values are presented by the myogenous factor - scale II $(0.27 \pm 0.26)$ and the by the psychoemotional factor - scale V $(0.28 \pm 0.06)$. Scale III (locoregional irradiation factor) and VII (pain intensity factor) have an intermediate position, due to their degree of expression $(0.13 \pm 0.06)$.

The components of the algic syndrome in patients with SB aged under 35 showed a pronounced expression for the following factors (in a decreasing order): myogenous ( $1.14 \pm 0.12)$, circadian $(0.99 \pm 0.11)$, psychoemotional $(0.92 \pm 0.11)$, pain duration $(0.58 \pm$ $0.08)$; a moderate expression for the following factors: pain intensity $(0.58 \pm 0.08)$, arthrogenic $(0.33 \pm 0.06)$; poor expression for the following factors: autonomous $(0.18 \pm 0.03)$, loco-regional irradiation $(0.16 \pm 0.05)$.

For the patients aged 35 years or older, the expression of the algic syndrome was characterized by pronounced psychoemotional manifestations $(0.83 \pm 0.13)$, circadian $(0.82 \pm 0.12)$, myogenous $(0.71 \pm 0.13)$, a longer duration $(0.66 \pm 0.11)$ and arthrogenic manifestations $(0.65 \pm 0.11)$; moderate for pain intensity $(0.32 \pm 0.02)$; poor expression for pain irradiation $(0.18 \pm 0.04)$ and autonomous manifestations $(0.16 \pm 0.05)$.

The analysis of the masseter muscle thickness was conducted based on the gender of the patients, because it was established that the differences of these indices are statistically significant between men and women $[18,19]$. During the state of relaxation, the masseter muscle thickness was $9.75 \pm 0.27 \mathrm{~mm}$ for women under 35 and of $9.82 \pm 0.26 \mathrm{~mm}$ for older women $(p>0.05)$. During jaw clenching, the values of masseter thickness were: $13.12 \pm 0.29 \mathrm{~mm}$ for younger women $(<35$ years, $\mathrm{n}=50)$, and for the ones over 35 $(n=18)-14.75 \pm 0.28 \mathrm{~mm}(p<0.001)$. With aging, the increase of the masseter muscle thickness was also evident.

In men, during relaxation, the thickness of the masseter muscle was $10.71 \pm 0.23 \mathrm{~mm}$ at the age of under 35 ( $\mathrm{n}$ $=20)$ and of $11.45 \pm 0.22 \mathrm{~mm}$ - over $35(\mathrm{n}=12, \mathrm{p}<$ $0.05)$; during jaw clenching, the thickness of the muscle was of $14.52 \pm 0.24 \mathrm{~mm}$ for individuals under 35 and of $16.21 \pm 0.28 \mathrm{~mm}$ for patients over $35(p<0.001)$.

As it may be observed from Table 2, the thickness gradient (relaxation-engagement) had a higher statistical significance in patients with $\mathrm{SB}$, which are over 35.

\section{Discussion}

According to the proposed objectives, we have studied the clinical peculiarities of SB, according to the age of the patients - under 35 and older. This division was made based on multiple literature data that reflects that the period of 35-40 years of age is the one where the prevalence of bruxism shows a considerable reduction [12].

Taking into account the fact that increased stress

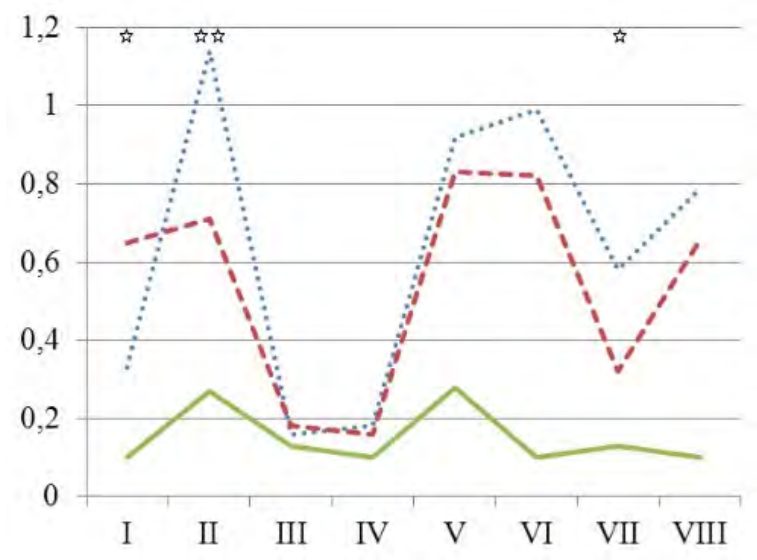

Figure 1. Bruxism-associated pain intensity in patients with primary sleep bruxism, according to the age of the patients.

Note: the scales (factors) of the bruxism-associated pain: I - arthrogenic; II - myogenous; III - loco-regional irradiation; IV - autonomous; V - psychoemotional; $\mathrm{VI}$ - circadian; VII - pain intensity; VIII - pain duration; statistical significant differences between sleep bruxism patients under 35 vs. patients over $35\left(^{*}-p<0.05,{ }^{*}-p<0.01\right)$. Blue dots - Sleep bruxism patients, under 35; Red dots - Sleep bruxism patients, over 35; Green line - Healthy subjects.

activity contributes to the occurrence of many SB disorders [26] and that people with SB have a much higher level of stress-sensitivity [27], we have analyzed the level of emotional stress (VAS) and the professions of the patients, according to the stress level.

We have observed that the level of emotional stress is an important index that is fundamentally different in healthy people and in patients with SB, but this index does not reflect the differences regarding the age of the investigated subjects, and some tendencies of increased stress levels in younger individuals can be observed.

With aging, there is an increase in the quantitative and qualitative sleep disruptions. The interpretation of these data is difficult due to the fact that, as it has been established that in healthy people, there are significant changes in the sleep quality associated with aging, especially after the age of 30-35 - a longer period of time is needed to get asleep, sleep is far more fragmented, with more frequent awakening episodes, the duration is shorter. These features are particularly greatly increased in patients with SB.

Pain in the masticatory muscles and in the temporomandibular joint is one of the main causes of dental visits [29]. For these reasons, a more precise clinical diagnosis of the algic syndrome will contribute to more effective monitoring and treatment.

The phenomenon of tongue ulcerations can be partially explained by the fact that the pressure exerted by the bruxer's tongue towards the teeth is much higher compared to the one found in non-bruxers [28]. It has been established that the bioelectric activity of the masticatory muscles may be associated with muscle pain but may also present as a disorder that is independent of the presence of pain and its intensity [29]. The relationship between pain and sleep bruxism is still a matter of controversy, especially in regard to whether a painful condition may affect the EMG activity during sleep [2]. Due to a massive nociceptive affinity from the structures of the stomatognathic 
system towards the somatosensory cortex, there occur various reflex activities, due to modifications of muscle tone and onset of myogenous pain. However, the correlations between reflex activity and pain expression are not yet studied deeply.

According to several research projects, there are no statistically significant differences in the EMG indices in patients, which feel pain in the masticatory muscles compared to those with no pain $[12,29]$. The authors state that this phenomenon is due to central neuronal adaptation mechanisms, modifications of the pain modulation mechanisms and because of central sensitization phenomena.

It should be taken into account that various poorly expressed algic manifestations may be present for a short term in healthy individuals as well.

It is to be noted that myogenous pain in younger patients is more pronounced than in older patients. This phenomenon was also observed by other researchers $[8,9,12,30,31]$, who explain it through the processes of adaptation to chronic pain observed in older people.

The results demonstrate that the psychoemotional factor (scale V), myogenous (scale II), circadian (scale $\mathrm{VI}$ ), and pain duration (scale VIII) are quite pronounced in SB patients, that are either under or over 35. The pain pattern in patients under 35 may be characterized as a psycho-emotionalmyogenous circadian pain and for the older patients as a psycho-emotional-myogenousarthrogenic circadian pain.

The data obtained confirm the results from the scientific literature - consequent to aging (until 61 years); an increase of the masseter muscle thickness can be observed [32].

There are several hypotheses regarding this problem: the increase of the masseter muscle thickness may occur as a result to muscle edema, fat accumulation, deficiency of protein metabolism, effects of psychoactive substances (alcohol, nicotine, etc.). Under the influence of previous inflammatory processes, the thickness of the muscle increases by approximately 4.3 $\mathrm{mm}$ [32].

Patients with SB show areas of muscle hypertonus (trigger zones) in which ultrasonographic examination shows changes in the echo-structure, left/right asymmetry and modification of the masseter muscle thickness.

In patients with SB under 35, the presence of local pathological echogenics is more frequent, which in most cases corresponds to the localization of the algic areas during palpation of the masseter muscles. In patients with SB over 35, besides the local pathological echogenicity, quite frequently a diffuse pathological echogenicity was observed. In the scientific literature, these aspects are being debated - it has been established that during the first stages of the disorders of the masseter muscle activity, local hypoechogenic areas appear, which afterwards, due to the aggravation of the disturbances, transform into diffuse hyperechogenic zones [18].

An important dental index for SB is the presence of dental abrasion. Surprisingly, there was no statistically significant correlations observed between the EMG activity of the muscles and the degree of dental abrasion [33]. Some researchers consider that currently, there is an overemphasis on SB as a causative factor in tooth wear [4]. No valid correlations were found between dental wear and the age of patients, the occlusal factors and the degree of temporomandibular joint dysfunction. These data demonstrate that wear is associated with various factors, including cerebral factors. Moreover, a specific study has not revealed any statistically significant differences in the bioelectric activity of masticatory muscles during sleep between patients with and without signs of dental wear [31]. While assessing dental abrasion as a diagnostic sign of SB, it is necessary to highlight other etiopathogenic factors; dental abrasion is not the main criterion in the diagnosis of SB, because it can occur in other pathologies as well (acid reflux, long-term consumption of acidic juices, etc.) [30,31].

In summary, the following SB manifestations occur with a higher severity in patients under 35 years of age (statistically significant), when compared to older patients $-p<0.05$ ):

- clinical signs of bruxism, assessed by means of the clinical questionnaire $(p<0,05)$;

- dysfunction of the temporomandibular joint, assessed by means of Fonseca questionnaire $(p<$ 0.05),

- total number of clenches (TNC) $(p<0.001)$,

- $\quad$ total duration of clenches (TCT) $(p<0.001)$.

The following SB manifestations occur with a higher severity in patients over 35 years of age (statistically significant), when compared to younger patients $-\mathrm{p}<$ 0.05):

- pathological thickness of the masseter muscle $(\mathrm{MM})$, determined by ultrasound $(\mathrm{p}<0.001)$,

pathological echogenicity of the $\mathrm{MM}$, determined by ultrasound $(p<0.01)$,

- $\quad$ shorter sleep duration $(p<0.05)$, pathological quality of sleep $(p<0.001)$.

The results obtained show that there are important peculiarities of the clinical manifestations of primary sleep bruxism according to the age of the patients, which can be detected by the application of clinical and paraclinical diagnostic methods, mainly by using technologies able to continuously monitor the bioelectric activity of the masticatory muscles and the morpho-functional status of the stomatognathic system through ultrasonography.

\section{Conclusions}

1. The expression of primary sleep bruxism is influenced by the age of the patients: the younger patients (18-35 years of age) are characterized by having more severe disorders regarding the episodes of sleep bruxism, with more pronounced myogenous-spastic manifestations; older patients (35-50 years of age) are characterized by the predominance of local and diffuse myogenicstructural disturbances, with more pronounced arthrogenic pains, with more severe quantitative and qualitative disturbances of nighttime sleep.

2. The expression of the emotional stress and the quantitative manifestations of dental abrasion are not essentially different in patients with primary sleep bruxism based on the age factor. 
3. The nighttime electromyography diagnosis via the application of portable devices and the ultrasound diagnosis can reveal important peculiarities of the activity of the masticatory muscles, regarding their local and diffuse thickness and echogenicity, associated with the severity of the primary sleep bruxism in patients of various age.

\section{Author Contributions}

Equal contribution to the paper.

\section{Acknowledgments}

Not applicable. The study was self-funded.

\section{References}

1. Yachida W, Arima T, Castrillon E, et al. Diagnostic validity of self-reported measures of sleep bruxism using an ambulatory single-channel EMG device. J Prosthodont Res. 2016;60(4):250257. doi: 10.1016/j.jpor.2016.01.001.

[Full text links] [Free Article] [PubMed] Google Scholar (4) Scopus (1)

2. Sateia MJ. International classification of sleep disorders-third edition: highlights and modifications. Chest. 2014;146(5):13871394. doi: 10.1378/chest.14-0970. Review.

[Full text links] [PubMed] Google Scholar (242) Scopus (106)

3. Croitoru C, Marinescu I, Drăghici E, et al. Etiological consideration in bruxism. Stoma Edu J. 2014;1(1):28-32. Google Scholar (0)

4. Jonsgar $C$, Hordvik PA, Berge $M$, et al. Sleep bruxism in individuals with and without attrition-type tooth wear: An exploratory matched case-control electromyographic study. J Dent. 2015;43(12):1504-1510. doi: 10.1016/j.jdent.2015.10.002 [Full text links] [Free Article] [PubMed] Google Scholar (11) Scopus (1)

5. Balatsouras D, Kaberos A, Psaltakos V, Papaliakos E, Economou N. Bruxism: two case reports. Acta Otorhinolaring Ital. 2004;24(3):165-170.

[PubMed] Google Scholar (9) Scopus (5)

6. Rao S, Bhat M, David J. Work, stress and diurnal bruxism: a pilot study among information technology professionals in Bangalore city, India. Int J Dent. 2011;2011:650489. doi: $10.1155 / 2011 / 650489$.

[Full text links] [Free PMC Article] [PubMed] Google Scholar (12) Scopus (6)

7. Oksenberg A, Aron SE. Sleep bruxism related to obstructive sleep apnea: the effect of continuous positive airway pressure. Sleep Med. 2003;3(6):513-515.

[Full text links] [PubMed] Google Scholar (119) Scopus (64)

8. Prasad DK, Swaminathan AA, Prasad DA. A review of current concepts in bruxism - diagnosis and management. NUJHS. 2014;4(4):129-136. Google Scholar (5)

9. Jain A, Bhaskar DJ, Yadav P, Lukram A, Khunrana R. Bruxism: an obscure pain. Int J Dent Med Res. 2014;1(1):21-30. Google Scholar (8)

10. Roopa G, Maansi BY, Shweta KM. Bruxism: prevalence among software professionals. IJCDS. 2011;2(2):69-73. Google Scholar (2)

11. de Freitas AR, Magalhães Dias $M$, Falcão Filho $H B L$, de Vasconcellos AA. Sleep bruxism in children: prevalence and multidisciplinary therapy. OHDM, 2014;13(4):897-901. Google Scholar (1)

12. Lavigne G, Tuomilehto H, Macaluso G. Pathophysiology of sleep bruxism. In: Lavigne GJ, Cistulli PA, Smith MT, Eds. Sleep medicine for dentists. A practical overview. Hanover Park, IL,USA: Quintessence Publishing Company, Inc.; 2009, pp. 117-124. Google Scholar (13)

13. Fonseca CM, dos Santos MB, Consani RL, dos Santos JF, Marchini $\mathrm{L}$. Incidence of sleep bruxism among children in Itanhandu, Brazil. Sleep Breath. 2011;15(2):215-220. doi: 10.1007/s11325010-0427-3.

[Full text links] [PubMed] Google Scholar (28) Scopus (13)

14. Kaushik SK, Madan R, Gambhir A, Prasanth T. Aviation stress and dental attrition. Int J Aerospace Med. 2009;53(1):6-10. Google Scholar (5)

15. Khoury S, Rouleau GA, Rompré $\mathrm{PH}$, et al. A significant increase in breathing amplitude precedes sleep bruxism. Chest. 2008;134(2):332-337. doi: 10.1378/chest.08-0115.
[Full text links] [PubMed] Google Scholar (58) Scopus (35)

16. Lobbezoo F, Ahlberg J, Manfredini D, Winocur E. Are bruxism and the bite causally related? J Oral Rehabil. 2012;39(7):489-501. doi: 10.1111/j.1365-2842.2012.02298.x. Review.

[Full text links] [PubMed] Google Scholar (75)

17. Vasconcelos PB, de Sousa LG, Regalo SC, et al. The influence of maxillary and mandibular osteoporosis on maximal bite force and thickness of masticatory muscles. Acta Odontol Latinoam. 2015;28(1):22-27. doi: 10.1590/S1852-48342015000100003. [Full text links] [Free Article] [PubMed] Google Scholar (3) Scopus (2)

18. Kiliaridis S, Mahboubi PH, Raadsheer MC, Katsaros C Ultrasonographic thickness of the masseter muscle in growing individuals with unilateral crossbite. Angle Orthod. 207:77(4):607-611. doi: 10.2319/101105-360.

[Full text links] [PubMed] Google Scholar (53) Scopus (26)

19. Najm AA. Sonographic evaluation of masseter muscle thickness in bruxist and non-bruxist subjects. J Bagh Coll Dent. 2014;26(3):49-52.

[PubMed] Google Scholar (2)

20. Rohila AK, Sharma VP, Shrivastav PK, Nagar A, Singh GP. An ultrasonographic evaluation of masseter muscle thickness in different dentofacial patterns. Indian J Dent Res. 2013;23(6):726731. doi: 10.4103/0970-9290.111247.

[Full text links] [Free full text] [PubMed] Google Scholar (11) Scopus (4)

21. Nishimura S. Work and ischemic heart disease. Japan Med Assoc J. 2004; 47(5):216-221.

[PubMed] Google Scholar (5)

22. Shetty S, Pitti V, Satish Babu CL, Surendra Kumar GP, Deepthi BC. Bruxism: a literature review. J Indian Prosthodont Soc 2010;10(3):141-148. doi: 10.1007/s13191-011-0041-5. [Full text links] [Free PMC Article] [PubMed] Google Scholar (101) Scopus (36)

23. Tosato J de P, Caria PH, Gomes CA, et al. Correlation of stress and muscle activity of patients with different degrees of temporomandibular disorder. J Phys Ther Sci. 2015; 27(4):12271231. doi: 10.1589/jpts.27.1227.

[Full text links] [Free PMC Article] [PubMed] Google Scholar (12) Scopus (4)

24. Woźniak K, Lipski M, Lichota D, Szyszka-Sommerfeld L. Muscle fatigue in the temporal and masseter muscles in patients with temporomandibular dysfunction. Bio Med Res Int. 2015;2015:269734. doi: 10.1155/2015/269734.

[Full text links] [Free PMC Article] [PubMed] Google Scholar (13) Scopus (6)

25. Casett E, Réus J, Stuginski-Barbosa J, et al. Validity of different tools to assess sleep bruxism: a meta-analysis. J Oral Rehabil. 2017;44(9):722-734. doi: 10.1111/joor.12520. Review. [Full text links] [PubMed] Google Scholar (1) Scopus (1)

26. Aloe F. Sleep bruxism neurobiology. Sleep Sci. 2009;2(1):40-48. Google Scholar (8) Scopus (3)

27. Bender SD. Sleep bruxism: a topical review. J Great Houst Dent Soc. 2012;5:10-14.

Google Scholar (3)

28. Meirelles L, Cunha Matheus Rodrigues Garcia R. Influence of bruxism and splint therapy on tongue pressure against teeth. Cranio. 2016;34(2):100-104. doi: 10.1179/2151090315Y.0000000010. [Full text links] [PubMed] Google Scholar (1) Scopus (0)

29. Mazzetto MO, Paiva G, Magri LV, Melchior MO, Rodrigues CA. Frequency of electromyographic indices alterations in temporomandibular disorders and their correlation with pain intensity. Ref Dor. 2014;15(2):91-95. doi: 10.5935/1806 0013.20140026. Google Scholar (2)

30. Klasser G, Rei N, Lavigne GJ. Sleep bruxism etiology: the evolution of a changing paradigm. J Can Dent Assoc. 2015;81:f2. Review.

[Full text links] [Free Article] [PubMed] Google Scholar (43) Scopus (14)

31. Veiga N, Ângelo T, Ribeiro O, Baptista A. Bruxism - literature review. Int J Dent Oral Health. 2015;1 (5):1-5. doi: 10.16966/23787090.134

Google Scholar (4)

32. Palinkas M, Nassar MS, Cecílio FA, et al. Age and gende influence on maximal bite force and masticatory muscles thickness. Arch Oral Biol. 2010;55(10):797-802. doi: 10.1016/j. archoralbio.2010.06.016

[Full text links] [PubMed] Google Scholar (118) Scopus (64)

33. Svensson $P$, Jadidi F, Arima T, Baad-Hansen L, Sessle BJ. Relationships between craniofacial pain and bruxism. J Oral Rehabil. 2008;35(7):524-547. doi: 10.1111/j.13652842.2008.01852.x. Review.

[Full text links] [PubMed] Google Scholar (161) Scopus (88) 


\title{
Dumitru ROMANIUC \\ PhD Fellow \\ Department of Therapeutic Dentistry, Faculty of Dentistry „Nicolae Testemițanu" State University of Medicine and Pharmacy \\ Chişinău, Republic of Moldova
}

Dr. Dumitru Romaniuc is a graduate of the State University of Medicine and Pharmacy "Nicolae Testemițanu" of the Republic of Moldova, Chisinau. From 2015, he is a PhD fellow at the "Nicolae Testemițanu" University of Medicine and Pharmacy. During his practice in general dentistry, he has published 28 articles and papers in national and international journals. He actively participates in various conferences and scientific meetings, both at the national level and abroad. He has obtained a gold medal at the Brussels Invention Fair (2015), a silver medal at the Geneva Invention Fair (2016), a gold medal at the Infolnvent exhibition (2017). He is the author of 3 patents in the domain of dentistry.

\section{Ouestions}

1. Based on epidemiological data, what percentage of the general population is affected by bruxism?

a. Up to $85-90 \%$;

b. Less than $0.1 \%$;

ac. Up to $20-30 \%$;

ad. Approximately $40 \%$.

\section{What age group is affected by bruxism?}
口a. Only adults;
bb. Mainly children;
ac. Only the geriatric population;
ad. All the age groups.

\section{Which of the following is not one of the bruxism cofactors?}
口a. Alcohol;
b. Caffeine;
b. Sleep;
ad. Nicotine.

\section{After the age of 65 , the prevalence of bruxism is:}

\author{
$\square$ a. Up to $1 \%$; \\ b. Up to $60 \%$, regardless of gender; \\ ac. The highest among the general population; \\ ad. Around $10 \%$ with a tendency to decrease in relation to age.
}

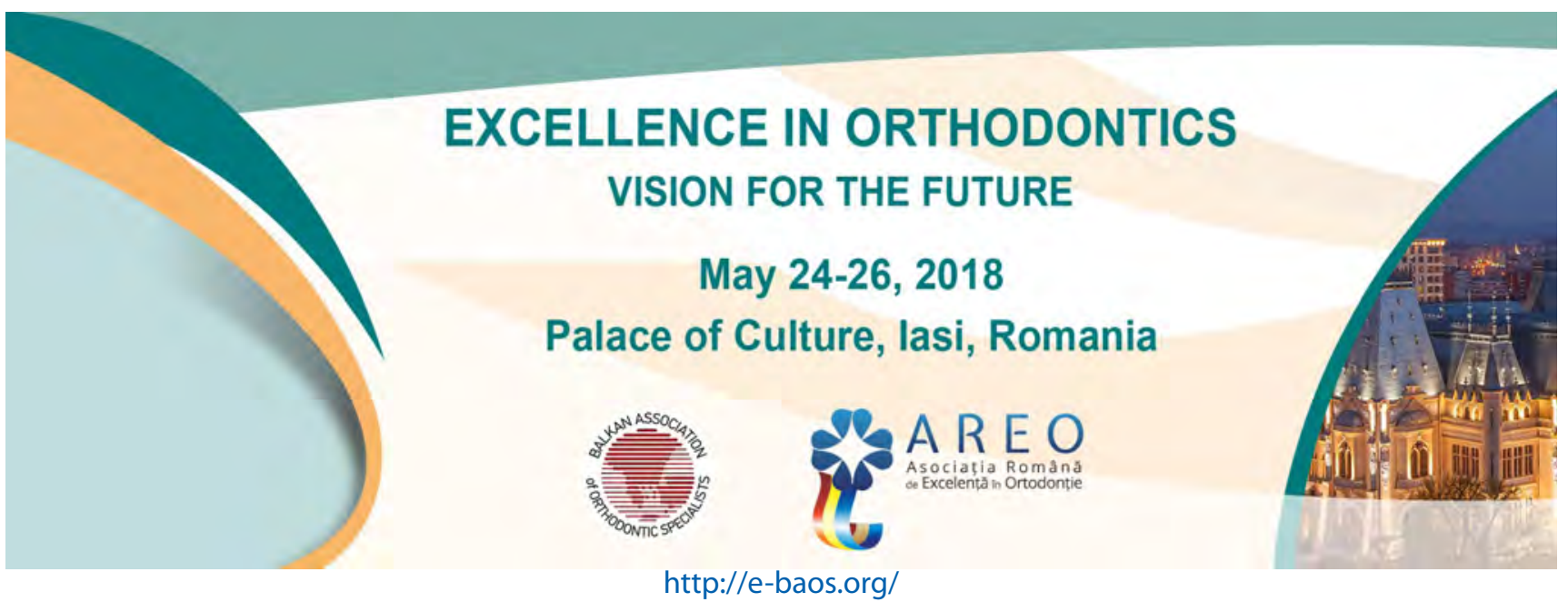

